Advances in Complex Systems

(c) World Scientific Publishing Company

\title{
MEASURING BRAND AWARENESS IN A RANDOM UTILITY MODEL
}

\author{
PIERFRANCESCO DOTTA \\ pierfrancesco.dotta@gmail.com \\ MARCO TOLOTTI \\ Department of Management, Ca' Foscari University of Venice, \\ Cannaregio 873, 30121 Venice, Italy \\ marco.tolotti@unive.it \\ JORGE YEPEZ \\ Department of Economics, Ca' Foscari University of Venice, \\ Cannaregio 873, 30121 Venice, Italy \\ jorge.yepez@unive.it \\ Received (09 12 2016) \\ Revised (revised date)
}

\begin{abstract}
Brand Awareness is recognized to be an important determinant in shaping the success of durables [16, 13], yet it is very difficult to be quantified. This is exactly the main goal of this paper: propose a suitable model where brand awareness of two competing firms is modelled and, eventually, estimated. To this aim, we build a random utility model for a duopoly where each competitor is characterized by different pricing strategies and brand awareness. As a result, different levels of market shares will emerge at the equilibrium. As a case study, we calibrate the model with real data from the smartphone industry obtaining an estimate of the value of the brand awareness of two leading brands.

Keywords: Random utility model; social interactions; discrete choice model; duopoly; brand awareness.
\end{abstract}

\section{Introduction}

Brand awareness plays an important role in consumer decision-making and is central on determining the success of companies. The objective of branding decisions in modern organizations is to generate a brand image for their products or services that is in line with the firm's target market and positioning decisions. Brand image is defined as the subjective perceptions and mental images that consumers' minds associates with a particular brand. Therefore, the branding process will result consumers recognizing and recalling the qualities of a particular brand with its image, a relation called brand awareness [7]. Brand awareness can be regarded as the degree in which consumers become familiar with a brand. A strong brand awareness 
is essential in order to form brand image, because when a brand is well established in the consumer's memory it is easier for the mental images and perceptions to be associated with a brand [2]. Joseph [8] and Keller [9] illustrate how a brand's image can be present in the mind of consumers and how it impacts their buying decisions. O'Cass and Siahtiri [11] argue that consumption behaviors characterize the desire to possess certain brands as a mean to achieve a particular status and self-fulfillment; therefore, branding plays an important role in order to project a certain image on potential customers and generate a positive brand awareness. For example, consumers buy a special clothes brand or car brand because they want to identify themselves with the brand and be associated with the prestige of the brand image [10].

A strong brand awareness and positve brand image also results in customers spreading brand loyalty and devotion: devoted consumers act to bring others' attention to the brand and attract new customers. In addition, potential adopters are attracted towards the group of consumers with the highest recognition [5] $]^{\mathrm{a}}$. Positive correlation was found between word of mouth, brand loyalty and brand awareness on luxury goods [15]. Summarizing, it is evident that social interactions play an important role in consumers buying decision process and brand awareness produces a positive externality, increasing in the company position on the market.

Our goal is to model brand awareness of two competitive firms in a society made of heterogeneous individuals with distinct preferences, where brand awareness plays a crucial role in the decision process. It produces a positive externality, which improves the company's position in the market. We micro found agents with heterogeneous preferences using a random utility model with a social component, in line with a traditional discrete social choice framework with social interactions. In their seminal paper [4], Brock and Durlauf propose a binary decision model where the action of single players is influenced by an aggregate signal represented by the (estimated) percentage of actors choosing one product or the other. In particular, actors are prone to imitate the behavior of the majority, thus, proving to be influenced by social interactions in their decision-making. In [12], the Brock and Durlauf paradigm is transferred into a game-theoretical language, thus highlighting the strategic behaviour of a large population of players subject to social interactions.

In the present paper, we extend the model in [12] to the case of a duopoly: two competitors, characterized by different levels of brand awareness and prices, offer a new technology on the market. These quantities enter as parameters into a wellposed random utility model and, eventually, shape the level of market shares for the two technologies at the equilibrium. As an illustrative example, we calibrate the

\footnotetext{
a As argued by Asch [1], "the primary mechanism in social influence is the change in the definition and meaning of an object". Woods and Hayes [16] tell us how the consumers perform a "social (re)construction of reality", reinterpreting the information about objects in relation to their reference groups, wondering whether this would be in line or not with their being, and thus leading to potential social reward or punishment.
} 
model using real data of the market share and the prices of the two major players in the smartphone industry: Apple and Samsung.

\section{A Duopoly and a Large Population of Possible Buyers}

Each agent in a large population of $N$ possible buyers has to decide among three mutually exclusive options: buying product $A$ from firm $A$, buying product $B$ from firm $B$ or stay out of the market. Each action yields a utility $U_{A}, U_{B}$ and $U_{0}$, respectively. The decision-making process can be visually represented by the decision tree depicted in Figure 1. ${ }^{\mathrm{b}}$

Starting from the left, the agent faces two subsequent decisions: $(i)$ to buy or not to buy a product, $(i i)$ whether to buy product $A$ or $B$. If $U_{0}>\max \left(U_{A}, U_{B}\right)$, we end up in Event 0 . Without loss of generality, we set $U_{0}=0$. On the other hand, as soon as the utility of buying product $A$ or $B$ is positive, the agent follows the higher branch and chooses the preferable outcome according to utilities $U_{A}$ and $U_{B}$.

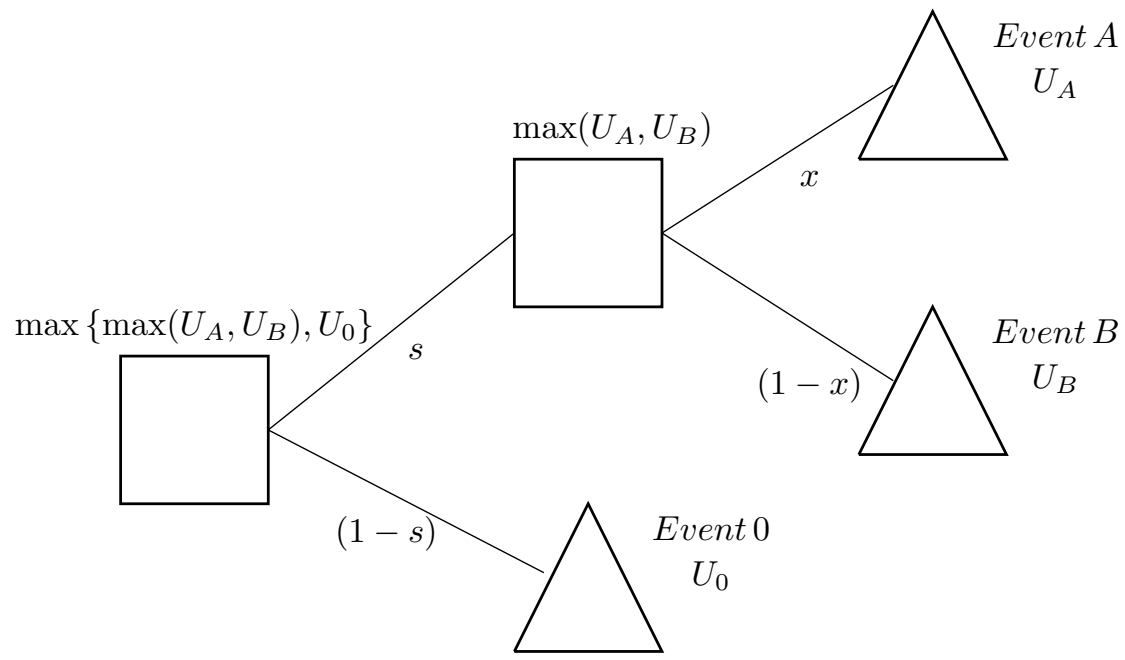

Fig. 1. Decision Tree representing to the decision process of the potential adopters.

Let us define $P_{A}=s \cdot x$ as the unconditional probability of buying $A$ and $P_{B}=s \cdot(1-x)$ the unconditional probability of buying $B$. Consequently, $s$ is

${ }^{\mathrm{b}}$ Square decision nodes indicate the moment at which the agent is required to take a decision, while triangles nodes are terminal nodes endowed with a utility value that the agent receives if the node is selected. The share of the population that decides to enter the market is $s$, while $x$ is the share of consumers in the market that purchase good $A$. 
the total probability of entering the market and $x$ is the probability of Event $A$ conditioned on the fact that the agent enters the market. In formulae

$$
\begin{gathered}
s=P_{A}+P_{B}=P\left(\max \left(U_{A}, U_{B}\right)>0\right), \\
x=\frac{P_{A}}{P_{A}+P_{B}}=P\left(U_{A}>U_{B} \mid \max \left(U_{A}, U_{B}\right)>0\right) .
\end{gathered}
$$

In order to compute the values of $s$ and $x$ at the equilibrium, we rely on a random utility model inspired by $[4,12]$. In detail, for each single actor $i=1, ., N$, we set

$$
\begin{gathered}
U_{A}(i)=-p_{A}+q_{A} x s+\epsilon_{A}(i), \\
U_{B}(i)=-p_{B}+q_{B}(1-x) s+\epsilon_{B}(i), \\
U_{0}(i)=0 .
\end{gathered}
$$

(3) and (4) resemble the standard shape of random utilities à la Brock and Durlauf (see [4]) and are composed of three terms. $p_{A}$ and $p_{B}$ are the market prices of technology $A$ and $B$ respectively; each of the second components introduces an externality due to social interactions. Indeed, $x s$ and $(1-x) s$ denote the respective market shares ${ }^{\mathrm{c}}$ prevailing at the equilibrium whereas $q_{A}$ and $q_{B}$ measure the level of brand awareness ${ }^{\mathrm{d}}$; finally, $\epsilon_{A}(i)$ and $\epsilon_{B}(i)$ are i.i.d. random variables introducing heterogeneity in the population of buyers ${ }^{\mathrm{e}}$. Therefore, they are all independent drawns from the same random variable $\epsilon$. Following standard literature in random utility models, we assume that $\epsilon_{A}(i)$ and $\epsilon_{B}(i)$ have a logistic probability distribution $\eta$ with mean zero and variance $\sigma^{2}=\frac{\pi^{2}}{3 \beta^{2}}$.

$$
\eta(z)=P(\epsilon \leq z)=\frac{1}{1-\exp (-\beta z)}, \quad \beta>0 .
$$

The bigger $\sigma^{2}$ (the smaller $\beta$ ) the more disperse the taste of the population of buyers.

Each agent compares among his utilities of adopting product $A$, product $B$, and not entering the market, $\left\{U_{A}(i), U_{B}(i), U_{0}\right\}$, as was described in Figure 1 . The agent's utilities when entering the market, $\left\{U_{A}(i), U_{B}(i)\right\}$, depend on the action of

${ }^{\mathrm{c}}$ The number of agents, $N$, can be partitioned into $N=N^{A}+N^{B}+N^{0}$. Consequently the market shares can be defined as $1=\frac{N^{A}}{N}+\frac{N^{B}}{N}+\frac{N^{0}}{N}$. Thus, when $N \rightarrow \infty, \lim _{N \rightarrow \infty} \frac{N^{0}}{N}=1-s$, $\lim _{N \rightarrow \infty} \frac{N^{A}}{N}=s x$ and $\lim _{N \rightarrow \infty} \frac{N^{A}}{N}=(1-x) s$.

$\mathrm{d}$ The coefficient multiplying the social component of the utility is interpreted as the force of externality or as the imitation driver (see for example [3], where a similar interpretation applies to the context of diffusion of innovation). In the same spirit, we interpret it here as the brand awareness of the issuing firm.

eProducts $A$ and $B$ are considered to have the same level of technology and quality. Consequently, the stochastic variables, $\epsilon_{A}$ and $\epsilon_{B}$, reflect the individual preferences for each good, hence the products are not vertically but horizontally differentiated. 
other agents through the participation shares shaping the social component term of the utilities. This eventually results in setting a non-cooperative game, in which each agent makes his choice given an expectation of the population outcome. Similarly to [12], agents do not communicate or coordinate, rather each individual knows the common distribution of the heterogeneous shocks $\epsilon_{j}$, for $j \neq i$. In other words, we impose rational expectations: each agent has a correct belief about others' preferences; moreover, we assume that each agent shares the same expectation about other player's actions ${ }^{\mathrm{f}}$. For a fixed population of $\mathrm{N}$ agents, where $N \rightarrow \infty$, at least one Nash equilibrium in pure strategies exists (see [6]). The result of this game theoretical setting -when we let $N \rightarrow \infty$ - is therefore the emergence of a Nash equilibrium characterized by levels $x^{*}$ and $s^{*}$. Because the logistic distribution is unimodal and S-shaped, we can have one or more equilibria depending on the set of prices $\left\{p_{A}, p_{B}\right\}$ and brand awareness $\left\{q_{A}, q_{B}\right\}$ (see [4]).

Under these assumptions, when the number of buyers tends to infinity, it is possible to derive an explicit expression for the probabilities $P_{A}$ and $P_{B}{ }^{\mathrm{g}}$ All these results are summarised in the next proposition:

Proposition 1. Assume a population of $N$ agents as described by equations (3)-(5) where $\left\{\beta, p_{A}, p_{B}, q_{A}, q_{B}\right\}$ are fixed and where $\eta_{A}$ and $\eta_{B}$ have the form (6). Then, at least one Nash equilibrium $\left(x_{N}^{*}, s_{N}^{*}\right)$ exists. Moreover, when $N \rightarrow \infty$,

$$
\left(x_{N}^{*}, s_{N}^{*}\right) \rightarrow\left(x^{*}, s^{*}\right)
$$

where $\left(x^{*}, s^{*}\right)$ solves the fixed point problem

$$
\left\{\begin{array}{l}
f(x, s)=0 \\
g(x, s)=0
\end{array}\right.
$$

with $f(x, s):=P_{A}+P_{B}-s$ and $g(x, s)=\frac{P_{A}}{P_{A}+P_{B}}-x$. Moreover,

$$
\begin{aligned}
P_{A}= & \frac{\exp \left(\beta X_{0}\right)}{\left(\exp \left(\beta X_{0}\right)-1\right)^{2}} \cdot \log \left(\frac{\exp \left(-\beta X_{0}\right)+\exp \left(\beta X_{A}\right)}{\exp \left(\beta X_{A}\right)+1}\right) \\
& +\frac{\exp \left(\beta X_{0}\right)}{\left(\exp \left(\beta X_{0}\right)-1\right)\left(\exp \left(\beta X_{A}\right)+1\right)}
\end{aligned}
$$

and

$$
\begin{aligned}
P_{B}= & \frac{-\exp \left(\beta X_{0}\right)}{\left(\exp \left(\beta X_{0}\right)-1\right)^{2}} \cdot \log \left(\frac{\exp \left(\beta X_{B}\right)+1}{\exp \left(\beta X_{0}\right)+\exp \left(\beta X_{B}\right)}\right) \\
& -\frac{1}{\left(\exp \left(\beta X_{0}\right)-1\right)\left(\exp \left(\beta X_{B}\right)+1\right)}
\end{aligned}
$$

where $X_{A}=p_{A}-q_{A} x s, X_{B}=p_{B}-q_{B}(1-x) s$ and $X_{0}=p_{B}-p_{A}-q_{B} s+\left(q_{A}+q_{B}\right) s x$.

${ }^{\mathrm{f}} \mathrm{We}$ are aware that our model is over simplifiying the micro structure behind the decision process of the agents. Information asymmetries, heterogeneous preferences, local interactions or network effects could be introduced; on the other hand, this would make the model much more complicated, causing a loss of tractability.

${ }^{\mathrm{g}}$ For a derivation, see the Appendix 
Note that the problem is intrinsically bi-dimensional in that $x$ and $s$ have to be determined at the equilibrium as the solutions to (7). Depending on the values of the parameters $\left\{\beta, q_{A}, q_{B}, p_{A}, p_{B}\right\}$, different equilibria emerge. Indeed, all the equilibrium solutions $\left(x^{*}, s^{*}\right)$ can be found by solving

$$
\left(x^{*}, s^{*}\right)=\underset{(x, s) \in[0,1] \times[0,1]}{\operatorname{argmin}}\{\phi(x, s)\},
$$

where

$$
\phi(x, s)=f(x, s)^{2}+g(x, s)^{2} .
$$

As an example, we run a simulation where we consider a fixed population (represented by $\beta=2$ ). In Figure 2 we plot the contour levels of equation (11), when firm A has a stronger brand awareness $\left(q_{A}=4, q_{B}=1\right)$, but the price of firm B is more competitive $\left(p_{A}=1.5, p_{B}=1\right)$. The black dots represent the solution points $\left(x^{*}, s^{*}\right)$ of $(10)$ depicted at points $(0.35,0.23),(0.66,0.38)$ and $(0.99,0.99)$. The top right corner displays the equilibrium point $(0.99,0.99)$, which illustrates the strong effect of brand awareness and social interaction, where firm 2 is practically taken out of market. Moreover, even at the most favourable equilibrium point for firm 2 , $(0.35,0.23)$, firm 1 still possesses an important market share and still competes on the market, although the total market share $(s)$ is considerably reduced.

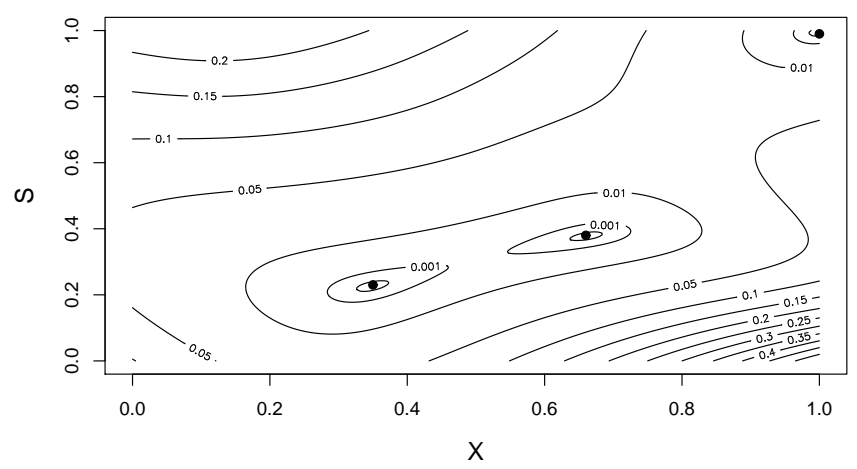

Fig. 2. Contour levels and equilibrium points $\left(x^{*}, s^{*}\right)$ of the function $\phi(x, s)$

In Table 1 we collect the values of $\left(x^{*}, s^{*}\right)$ emerging at the equilibrium for different specifications of the parameters. The first row shows a baseline full symmetric situation where the two firms share equal characteristics and where three equilibria 
Table 1. Equilibrium values of $(x, s)$ for different values of the parameters.

\begin{tabular}{|l|l|l|l||c|}
\hline \multicolumn{4}{|c|}{ Parameters } & Equilibrium Points \\
\hline \hline$q_{A}$ & $q_{B}$ & $p_{A}$ & $p_{B}$ & $\left(x^{*}, s^{*}\right)$ \\
\hline 2 & 2 & 0.5 & 0.5 & $(0.90,0.95),(0.50,0.91),(0.10,0.95)$ \\
\hline 2 & 2 & 1 & 0.5 & $(0.04,0.94)$ \\
\hline 4 & 2 & 0.5 & 0.5 & $(1.00,1.00)$ \\
\hline 4 & 2 & 1.5 & 0.5 & $(0.99,0.99),(0.54,0.9),(0.01,0.94)$ \\
\hline 1 & 1 & 1 & 1 & $(0.50,0.28)$ \\
\hline 4 & 1 & 1.5 & 1 & $(0.99,0.99),(0.66,0.38),(0.35,0.23)$ \\
\hline
\end{tabular}

emerge $^{\mathrm{h}}$. When $p_{A}$ increases (second row), there is only one equilibrium, at which the market share of product $B$ is dominant, when $q_{A}$ increases (third row) the market share of product $A$ is dominant. The fourth row shows how the negative effect of $p_{A}$ can be attenuated when $q_{A}$ is high. Additionally, we can see that when brand devotion is high, the total market size $s$ increases. The fifth row shows a scenario similar to the first one, where both products share equal characteristics; however, the prices are twice higher, yet brand awareness is cut by half. In this case the positive effect of brand awareness is not strong enough to offset the negative effect of higher prices. Consequently, there is only one equilibrium where firms share equal market share but where the total market size is considerably reduced. Finally, the last row shows exactly the scenario depicted on Figure 2, which is similar to row 4, except that $p_{B}$ is higher and $q_{B}$ is lower. As a result, both the medium equilibrium and the one where product $B$ is dominant show a smaller total market size, as well a stronger market share for firm $A$.

\section{A Case Study: The Smartphone Industry}

We apply our model to the case of the smartphone industry. We used the Gartner iDC data ${ }^{\mathrm{i}}$ and extract quarterly market shares (Q4 2012-Q4 2014), illustrated in the following figure:

\footnotetext{
${ }^{\mathrm{h}}$ The presence of multiple equilibria is due to the non-linearity of the system (7) and has significant consequences on the strategic behavior of firms (see, for instance, [12]). In standard random utility models, it is shown that for $\left\{q_{A}, q_{B}\right\}$ large enough, multiple equilibria may appear. In our setting, the picture is less clear because of the presence of the two competitors. For the sake of brevity, we leave this issue to future further investigation.

${ }^{\mathrm{i}}$ Data taken from Gartner, iDC at http://www.gartner.com
} 


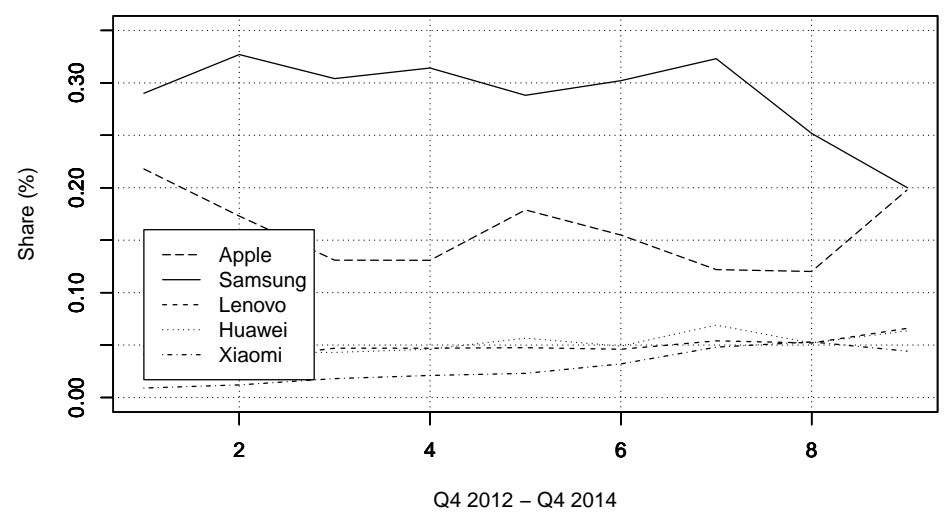

Fig. 3. Smartphone manufacturer industry market share, taken from Gartner iDC.

Looking at Figure 3, representing the market shares of the major competitors in the phone industry, it is easy to spot the predominance of the Samsung-Apple duo, holding under their control $40 \%$ of the global market, with their best performing competitors lagging more than 13 percentage points behind. For this reason and for the importance that socio-psychological dynamics assumed in the choice of our next smartphone, we believe that the smartphone industry represents a perfect case study to test the model being presented.

Samsung and Apple have adopted different strategies when it comes to their offered portfolio of products. Apple offers a small variety of mobiles phones compared to the considerably wider collection of products offered by Samsung. In our analysis, we focus on the S5 and S6 Samsung's smartphones, and the iPhone 6 family plus the iPhone 5S Apple's collection. ${ }^{\mathrm{j}}$ The average price $^{\mathrm{k}}$ for a Samsung model is $€ 550$, while for Apple's is $€ 729$. We let Apple represent product $A$ and Samsung product $B$. Being the model of comparative nature, we normalize $p_{B}=1$ and $q_{B}=2 .{ }^{1}$ Then, we set $p_{A}=\frac{729}{550}=1.325$. Finally, $q_{A}$ and $\beta$ will be calibrated. To this aim, we rely

jTaking into account the product's release date and Apple's higher prices, the Samsung's S5 and S6 in all of their different versions represent the main competitors of Apple's products.

kSamsung: S6 Edge Plus (€839), S6 Edge (€739), S6 (€739), S5 Neo (€330), S5 (€410), S5 Mini $(€ 240)$. Apple: iPhone 6S Plus (€779), iPhone 6S (€889), iPhone 6 Plus (€779), iPhone 6 (€669), iPhone 5S (€529).

${ }^{1}$ The value of $q_{B}=2$ has been chosen after a careful preprocessing of the model. We have tested it using $q_{B}$ set equal to $\{1,2,3,4\}$. When $q_{B}=1, q_{A}^{*}$ results to be negative (not feasible); while when $q_{B}>2$, the value of $\beta^{*}$ falls between 5 and 50 which we considered too high. The parameter $\beta$ is interpreted as a measure of the variance $\left(\sigma^{2}=\frac{\pi^{2}}{3 \beta^{2}}\right)$; therefore, values between $[5,50]$ imply that the population preferences are not heterogeneously enough distributed but the probability mass is concentrated around zero, with a variance between [0.1,0.001]. 
on equation (11), taking $s$ and $x$ as suggested from the quarterly market shares (Q4 2012-Q4 2014) of the smartphone industry. Figure 4 shows the estimated brand awareness ratio $q_{A}^{*} / q_{B}$, for the different quarters under analysis.

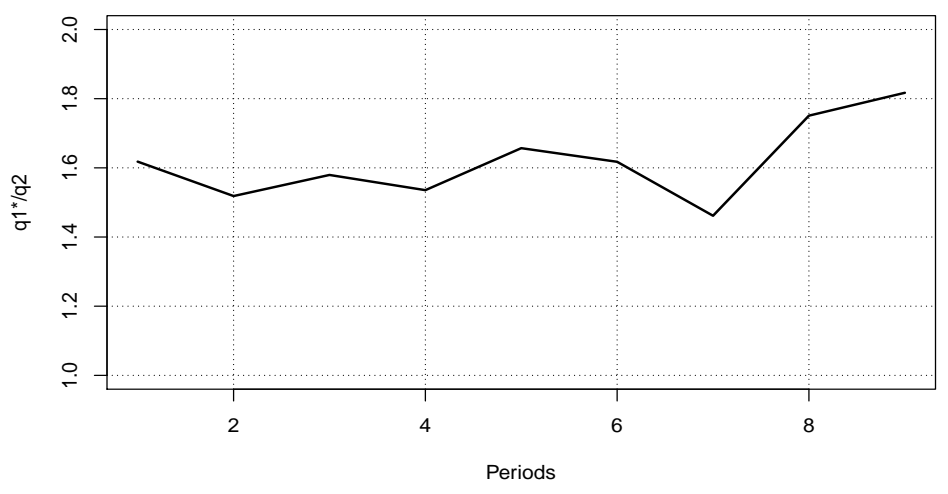

Fig. 4. Estimated brand awareness $q_{A}^{*} / q_{B}$, when $q_{B}=2$.

Our model shows a consistent higher level of Apple brand awareness compared to Samsung. Brand awareness and brand image has a significant impact on customer behavior [17]. Moreover, brand image is perceived as an important driving force of customer loyalty [14]. It is no hyperbole stating that Apple consumers make religious references to Steve Jobs and Apple's products, to the point that some of them are used to queue for days waiting for the release of the latest iPhone. Our model thus corroborates the well-known fact that the brand awareness of Apple is higher than the one of other firms. The novelty brought about by this model consists in its capacity to provide estimates of the ratio between the two main competitors' brand awareness; moreover, the values we find are consistent with price and market shares data. The maximum points of the ratio of Apple's to Samsung's awareness degree corresponds to the quarters in which Apple introduced its new products on the market: i.e., period 5 (Q4-2013) and 9 (Q4-2014). The decline after the fifth period could reflect the disappointment of the consumers who would have preferred to see a completely new smartphone, instead of an updated version of the old one (i.e., the iPhone 5S Apple release). Such a result shows that no matter how strong Apple's brand is, there is still a chance for other brands to capture a slice of its market share. Finally, our calibration exercise also provides an estimate $\sigma^{2}=1^{\mathrm{m}}$ for the variance of the logistic distribution characterizing the spread of taste among

${ }^{\mathrm{m}}$ The average estimated $\beta^{*}$ across the evaluated periods is 1.81 . 
the buyers. This figure, often used in random utility models, is rarely calibrated to real data.

\section{Final Remarks}

Social interaction plays an important role in order to determine the success of goods or services, specially when consumers react differently to brand images that firms promote to capture their attention $[1,15,16]$. In this paper we have modelled consumer decision making under the assumption that brand awareness plays the role of a social component in the utility. We suggest a different way to look at and use random utility models. A large number of agents faces two subsequent choices: adopt or not a new technology and, eventually, which one between the two releases of the technology to buy. Dealing with a duopoly, a peculiarity of our model is the presence of two bunches of parameters characterizing the two competitors. Once provided the equations needed to determine the market shares at the equilibrium, we calibrate the model with real data related to the major players on the smartphones industry, obtaining a quantitative measure of the brand awareness ratio of the two competitors. Our results support the fact that Apple's brand awareness is higher than the one of other firms.

\section{References}

[1] Asch, S., Social Psychology (Prentice-Hall, Englewood Cliffs, 1952).

[2] Esch, F., Langner, T., Schmitt, B., Geus, P., Are brands forever? How brand knowledge and relationships affect current and future purchases, Journal of Product 8 Brand Management 15 (2006) 98-105.

[3] Bass, Frank M., A New Product Growth for Model Consumer Durables, Management Science 15 (1969) 215-227.

[4] Brock, W. A. and Durlauf, S. N., Discrete Choice with Social Interactions, The Review of Economic Studies 68 (2001) 235-260.

[5] Chung, E., Beverland, M., Farrelly, F., and Quester, P., Exploring consumer fanaticism: Extraordinary devotion in the consumption context, Advances In Consumer Research [P] 35 (2008) 333-340.

[6] Dai Pra, P., Sartori, E., and Tolotti, M., Strategic Interaction in Trend-Driven Dynamics, Journal of Statistical Physics textbf152 (2013) 724-741.

[7] Drummond, G. and Ensor, J., Introduction to Marketing Concepts (Routledge Oxford; Boston, MA, 2005).

[8] Joseph, J., How Do I Love Thee, Apple? Let Me Count the Ways, Brandweek (2010) (3030).

[9] Keller, K., Strategic Brand Management: Global Edition (Pearson Education Limited, 2013).

[10] McColl, J. and Christopher, M., An exploration of fashion retailer own brand strategies, Journal of Fashion Marketing and Management: An International Journal 15 (2011) 91-107.

[11] O'Cass, A. and Siahtiri, V., In search of status through brands from Western and Asian origins: Examining the changing face of fashion clothing consumption in Chinese young adults, Journal of Retailing and Consumer Services 20 (2013) 505-515. 
[12] Pellizzari, P., Sartori, E., and Tolotti, M., Trade-In Programs in the Context of Technological Innovation with Herding, in Advances in Artificial Economics, eds. Amblard, F., Miguel, F. J., Blanchet, A., and Gaudou, B., number 676 in Lecture Notes in Economics and Mathematical Systems (Springer International Publishing, 2015)

[13] Reimann, M., Castano, R, Zaichkowsky, J., and Bechara, A., How we relate to brands: Psychological and neurophysiological insights into consumerbrand relationships, Journal of Consumer Psychology 22 (2012) 128-142.

[14] Saeed, R., Nawaz, R., Mehmood A., Ishfaque, U., Fareha, D., Amna, S., Zahid, M. and Moeed, A., Effect of Brand Image on Brand Loyalty and Role of Customer Satisfaction in it, World Applied Sciences Journal 26 (2013) 1364-1370.

[15] Virvilaite, R., Tumasonyte, D., and Sliburyte, L., The Influence of Word of Mouth Communication on Brand Equity: Receiver Perspectives, Procedia - Social and Behavioral Sciences 213 (2015) 641-646.

[16] Wood, W. and Hayes, T., Social Influence on consumer decisions: Motives, modes, and consequences, Journal of Consumer Psychology 22 (2012) 324-328.

[17] Zhang, Y., The Impact of Brand Image on Consumer Behavior: A Literature Review, Open Journal of Business and Management 3 (2015) 58-62

\section{Appendix A. Proof of Proposition 1}

Proof. The existence of Nash equilibria and the convergence of $\left(x_{N}^{*}, s_{N}^{*}\right)$ to the points solving $(7)$ can be deduced from arguments developed in [6]. We now concentrate on the shape of the functions $f(x, s)$ and $g(x, s)$, hence on $P_{A}$ and $P_{B}$. From (3) and (4), it follows that

$$
\begin{gathered}
P\left(U_{A}(i)>0\right)=P\left(-p_{A}+q_{A} x s+\epsilon_{A}(i)>0\right)=P\left(\epsilon_{A}(i)>X_{A}\right) \\
P\left(U_{B}(i)>0\right)=P\left(-p_{B}+q_{B}(1-x) s+\epsilon_{B}(i)>0\right)=P\left(\epsilon_{B}(i)>X_{B}\right)
\end{gathered}
$$

where $X_{A}=p_{A}-q_{A} x s, X_{B}=p_{B}-q_{B}(1-x) s$. Therefore, (8) can be derived as follows:

$$
\begin{aligned}
P_{A}=P\left(U_{A}(i)>U_{B}(i), U_{A}(i)>0\right)= & P\left(\epsilon_{B}(i)-\epsilon_{A}(i)<X_{0}, \epsilon_{A}(i)>X_{A}\right) \\
= & P\left(\epsilon_{B}(i)<X_{0}+\epsilon_{A}(i), \epsilon_{A}(i)>X_{A}\right) \\
= & \int_{X_{A}}^{\infty} \eta\left(X_{0}+\xi\right) d \eta(\xi) \\
= & \int_{X_{A}}^{\infty}\left(\frac{1}{1+\exp \left(-\beta\left(X_{0}+\xi\right)\right)}\right) \frac{\beta \exp (-\beta \xi)}{(1+\beta \exp (-\beta \xi))^{2}} d \xi \\
= & \int_{X_{A}}^{\infty} \frac{\beta}{\left(\exp (-\beta \xi)+\exp \left(-\beta X_{0}\right)\right)(1+\exp (-\beta \xi))^{2}} d \xi \\
= & \frac{\exp \left(\beta X_{0}\right)}{\left(\exp \left(\beta X_{0}\right)-1\right)^{2}} \cdot \log \left(\frac{\exp \left(-\beta X_{0}\right)+\exp \left(\beta X_{A}\right)}{\exp \left(\beta X_{A}\right)+1}\right) \\
& \quad+\frac{\exp \left(\beta X_{0}\right)}{\left(\exp \left(\beta X_{0}\right)-1\right)\left(\exp \left(\beta X_{A}\right)+1\right)},
\end{aligned}
$$

where $X_{0}=p_{B}-p_{A}-q_{B} s+\left(q_{A}+q_{B}\right) s x$. 
We used the convolution formula for independent random variables to derive the third line and the form of the logistic distributions of $\eta_{A}$ and $\eta_{B}$ to derive the fourth. The latter expression follows by direct integration: differently from the normal random variable, an explicit expression for the logistic distribution can be provided.

Similarly, (9) is derived as follows:

$$
\begin{aligned}
P\left(U_{B}(i)>U_{A}(i), U_{B}(i)>0\right) & =P\left(\epsilon_{B}(i)-\epsilon_{A}(i)>X_{0}, \epsilon_{B}(i)>X_{B}\right) \\
& =P\left(\epsilon_{A}(i)<\epsilon_{B}-X_{0}(i), \epsilon_{B}(i)>X_{B}\right) \\
& =\int_{X_{B}}^{\infty} \eta\left(\xi-X_{0}\right) d \eta(\xi) \\
& =\int_{X_{B}}^{\infty}\left(\frac{1}{1+\exp \left(-\beta\left(\xi-X_{0}\right)\right)}\right) \frac{\beta \exp (-\beta \xi)}{(1+\beta \exp (-\beta \xi))^{2}} d \xi \\
& =\int_{X_{B}}^{\infty} \frac{\beta}{\left(\exp (\beta \xi)+\exp \left(\beta X_{0}\right)\right)(1+\exp (-\beta \xi))^{2}} d \xi \\
= & \frac{-\exp \left(\beta X_{0}\right)}{\left(\exp \left(\beta X_{0}\right)-1\right)^{2}} \cdot \log \left(\frac{\exp \left(\beta X_{B}\right)+1}{\exp \left(\beta X_{0}\right)+\exp \left(\beta X_{B}\right)}\right) \\
& -\frac{1}{\left(\exp \left(\beta X_{0}\right)-1\right)\left(\exp \left(\beta X_{B}\right)+1\right)} .
\end{aligned}
$$

